\title{
Atresia de coanas en la edad adulta
}

\author{
Choanal atresia in adults
}

Rodolfo Nazar $\mathrm{S}^{1}$, Alfredo Naser $\mathrm{G}^{1}$, Juan Fullá $0^{2}$.

\begin{abstract}
RESUMEN
La atresia de coanas $(A C)$, se define como la falta de conexión entre la cavidad nasal y el tracto aerodigestivo, produciendo obstrucción parcial o total de una o ambas coanas. La etiología más frecuente es congénita, y muchas veces se presenta asociada con otras malformaciones. La causa adquirida es menos frecuente, se han reportado casos en pacientes que han requerido el uso de sondas por vía nasal por periodos prolongados. A continuación se presenta el caso clínico de una paciente de 44 años con el diagnóstico de atresia de coanas adquirida, quien tiene el antecedente de uso prolongado de sonda nasoyeyunal.
\end{abstract}

Palabras clave: Atresia coanal, malformación nasofaríngea

\begin{abstract}
Choanal atresia ( $A C$ ) is defined as the lack of connection between the nasal cavity and the aerodigestive tract, resulting in partial or total obstruction of one or both choanae. The most frequent etiology is congenital, and in many cases it is associated to other malformations. Acquired choanal atresia is less frequent; although cases have been reported in which patients had required nasal tubing for prolonged periods of time. The clinical case of a 44 year-old female diagnosed with acquired choanal atresia is presented here. The patient had a history of prolonged use of a nasojejunal feeding tube.

Key words: Choanal atresia, nasal obstrucction
\end{abstract}

Médico Otorrinolaringólogo, Hospital Clínico de la Universidad de Chile.

2 Interno de Medicina sede Norte de la Universidad de Chile. 


\section{INTRODUCCIÓN}

La arquitectura nasal empieza a estructurarse desde la cuarta hasta la duodécima semana de vida intrauterina. Se postula que la atresia congénita de coanas se produciría entre la sexta y la novena semana, debido a la persistencia de la membrana buconasal 0 a la persistencia de células epiteliales ${ }^{1}$. Constituye una de las alteraciones del desarrollo nasal más frecuente y cuando su compromiso es bilateral puede causar la muerte del recién nacido por asfixia, constituyendo una de las pocas urgencias otorrinolaringológicas en el período neonatal.

En relación a su adquisición en la edad adulta se ha descrito en pacientes con uso prolongado de sondas, pacientes con antecedentes de cirugías otorrinolaringológicas previas (posterior a una adenoidectomía), pacientes con cicatrización queloídea y en pacientes con carcinoma nasofaríngeo tras ser sometidos a radioterapia. La explicación sería la formación de un tejido fibroso, llegando a formar una verdadera capa membranosa que ocluye parcial o totalmente a una 0 ambas coanas ${ }^{2}$.

\section{CASO CLÍNICO}

Se presenta el caso clínico de una paciente mujer, de 44 años de edad, que dentro de sus antecedentes previos destacan, una rinitis alérgica y un intento de suicidio en el año 1990, a raíz del cual requirió la instalación de una sonda nasoyeyunal por 2 meses. En el año 2005 consulta al Servicio de Otorrinolaringología del Hospital Clínico de la Universidad de Chile por un cuadro de 2 años de obstrucción nasal bilateral progresiva, descarga posterior, rinorrea, hiponasalidad e hiposmia.

Al examen físico se aprecia rinorrea serosa, una mucosa nasal de aspecto pálido y una desviación septal a izquierda, planteándose como diagnósticos una desviación del tabique nasal y una rinosinusitis crónica.

Se inicia tratamiento con corticoides intranasales y se solicita tomografía axial computada de cavidades paranasales (Figuras 1, 2 y 3 ) que muestra cambios inflamatorios con discreto engrosamiento mucoso maxilar bilateral, estenosis progresiva de ambas fosas nasales, obliteración de las coanas por densidad de partes blandas y una desviación septal posterior izquierda. Frente a estos hallazgos se realiza una endoscopía nasal rígida, en la que se observa a nivel de la fosa nasal derecha, una estenosis significativa de la coana y una sinequia desde el cornete medio al tabique nasal. Al examinar la fosa nasal izquierda, se aprecia una desviación septal a izquierda y una sinequia desde el cornete medio al tabique nasal. Por tales hallazgos se plantean como diagnósticos definitivos una desviación septal izquierda y una estenosis coanal bilateral, sometiendo a la paciente a una septoplastía y una resección endoscópica de la estenosis.

Durante la cirugía endoscópica se encontró una estenosis coanal membranosa, tomándose una muestra del tejido para ser estudiado. El resultado diferido de la biopsia mostró la presencia de

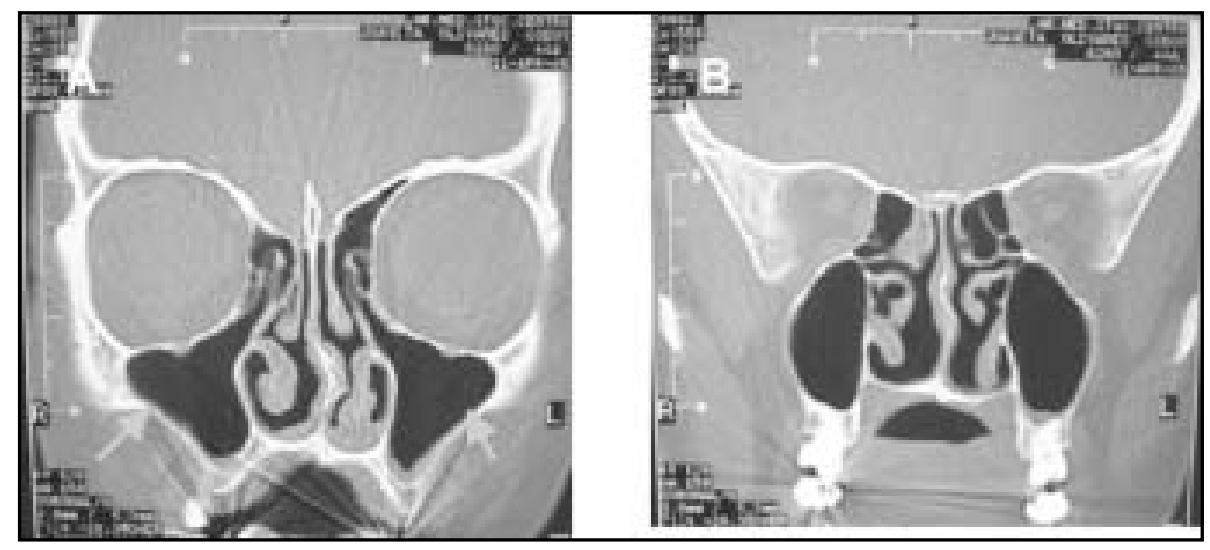

Figura 1. Lámina A y B. Cortes coronales de TAC de cavidades paranasales que muestra cambios inflamatorios con discreto engrosamiento mucoso maxilar bilateral. 


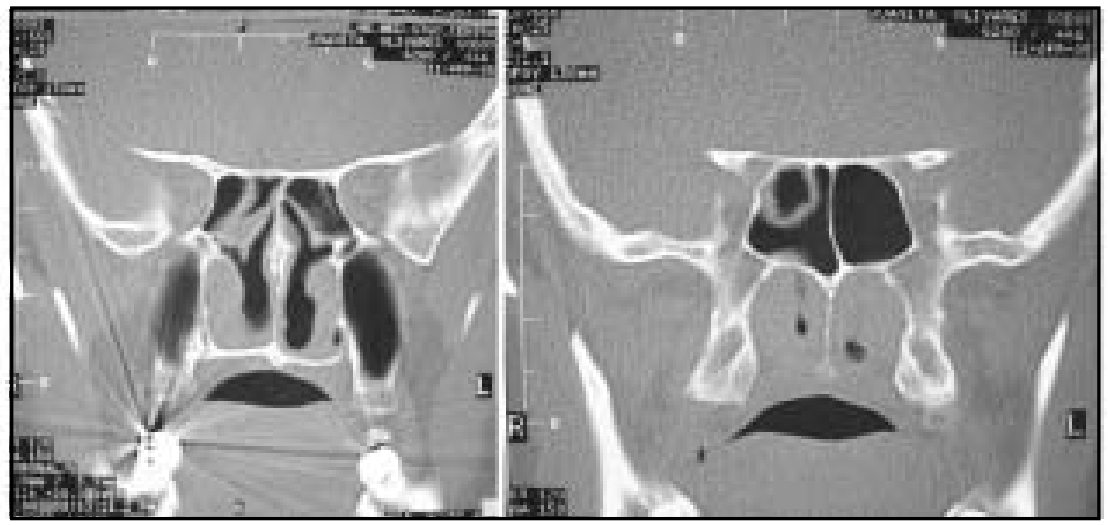

Figura 2. Lámina A y B. Cortes coronales de TAC de cavidades paranasales que muestra estenosis progresivas de ambas fosas nasales y obliteración de las coanas por partes blandas.

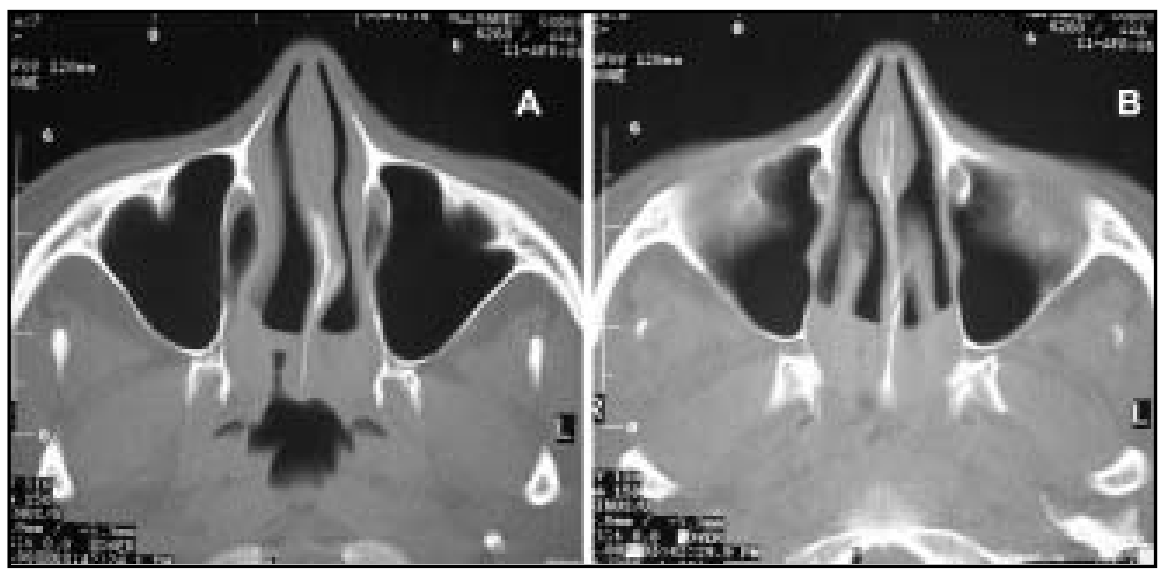

Figura 3. Lámina A y B. Cortes axiales de TAC de cavidades paranasales. Se observa una septodesviación posterior izquierda y obliteración de coanas por densidad de partes blandas.

abundante tejido inflamatorio crónico inespecífico y extensa fibrosis.

Se confeccionó un colgajo mucoso a 3-5 mm preestenosis a nivel coanal, removiendo en su totalidad un anillo grueso y fibroso, tratando de conservar el máximo de mucosa sana para recubrir el tejido óseo que quedó expuesto, lográndose permeabilizar ambas coanas. También se resecó la parte posterior del tabique nasal, produciendo una mayor permeabilización entre las fosas nasales y la rinofaringe (Figura 4). Se instaló un tutor de silicona bilateral (Figura 5) por 8 semanas, con buena tolerancia. Durante los controles realizados a las $2 y$ 3 semanas, se observaron costras intranasales. En la coana que cubría el torus tubario se realizó un mejor control de costras con aseo, solución fisiológica, uso de gentamicina, evolucionando con buena respuesta tanto de sus costras como de la otitis serosa. La paciente se mantuvo con aseo de solución salina por un período de un año sin presentar otras molestias salvo leves costras nasales.

El control endoscópico a los 18 meses de seguimiento, mostró permeabilidad coanal bilateral (Figura 6).

\section{DISCUSIÓN}

La primera descripción de atresia de coanas (AC) fue hecha por Johann Roederer en el año 1755, 


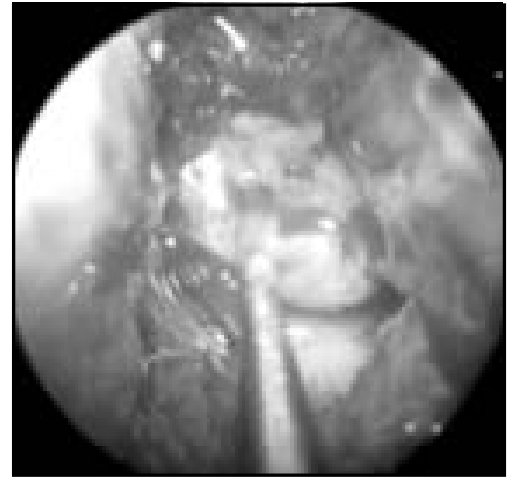

Figura 4. Confección de colgajo mucoso, durante la cirugía endoscópica funcional derecha que permeabilizó la fosa nasal derecha.

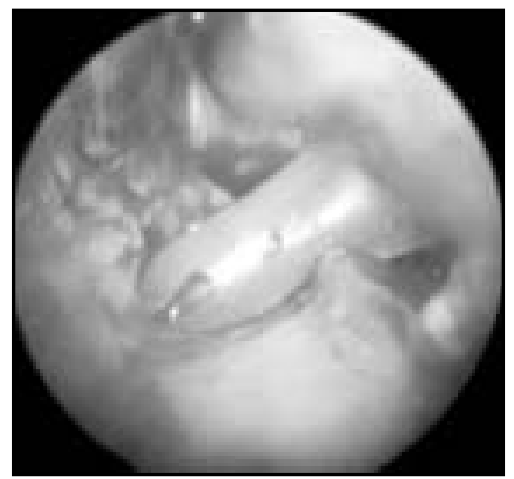

Figura 5. Colocación de Stent, por la fosa nasal en forma de U.

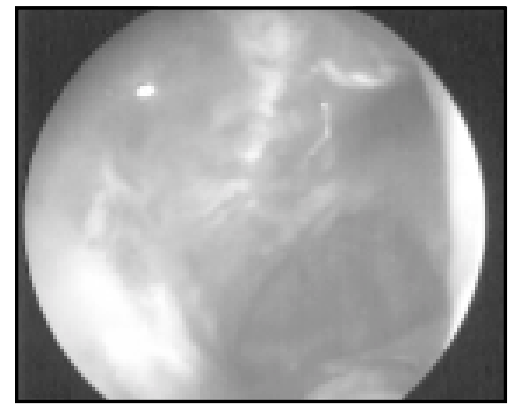

Figura 6. Imagen posoperatoria que muestra permeabilidad de la coana derecha.

durante la evolución clínica de un neonato. Fue hasta 1854 cuando Emmert, un pionero en la corrección quirúrgica de esta enfermedad, presentó un caso que respondió satisfactoriamente tras la introducción de un trócar curvo transnasal. ${ }^{3}$
Esta malformación congénita, consiste en la falta de conexión entre la cavidad nasal y el tracto aerodigestivo, y que produce obstrucción parcial 0 total de una 0 ambas coanas. En relación al sexo, en la literatura se describe una relación de dos mujeres por cada hombre afectado $0^{4,5}$.

La AC congénita suele presentarse en conjunto a otras malformaciones, esta asociación supera el $50 \%$. Está relacionado con la sigla CHARGE que deriva del inglés, correspondiendo cada letra a las seis mayores categorías involucradas: Coloboma $(C)$, enfermedad cardíaca $(H)$, atresia de coanas (A), retardo del desarrollo del sistema nervioso central $(R)$, genitourinarias $(G)$ y anomalías de oído o sordera $(E)$. Este término se utiliza cuando están presentes al menos cuatro de estas seis categorías ${ }^{1,3,4,6}$.

La AC se presenta de forma uni o bilateral, es decir, si están comprometidos uno 0 ambos lados de las fosas nasales. También puede ser ósea 0 membranosa, dependiendo del tejido comprometido, siendo en la mayoría de los casos (70\%) mixta, es decir mostrando un compromiso óseo y membranoso. En investigaciones recientes realizadas mediante tomografía computada y estudios histopatológicos, la afectación ósea se describe en el $30 \%$ de los pacientes, no encontrándose la existencia de obstrucción membranosa pura ${ }^{2,4,9}$.

La forma unilateral se observa más frecuentemente $(55 \%)$, sospechándose frente al antecedente de rinorrea purulenta unilateral. El compromiso bilateral constituye una verdadera emergencia al momento de nacer, ya que por estar el neonato obligado a respirar por vía nasal, presenta apnea, lo que obliga a asegurar la vía aérea mediante la colocación de un tubo endotraqueal, una cánula de Guedel o el chupete de Mc Govern',3.

Existen múltiples teorías que han intentado explicar el desarrollo de la atresia y su diferencia con la estenosis 0 atresia incompleta. Entre las causas congénitas destacan la persistencia de la membrana nasobucal de Hochtetter, la membrana bucofaríngea del intestino posterior y del tejido mesodérmico en forma de adherencias en la región coanal. Además, se han descrito alteraciones a nivel de la migración de las células mesodérmicas durante el desarrollo del embrión, y un crecimiento alterado en dirección medial, de los procesos vertical y horizontal del palatino $0^{4}$ (Figura 7 ). 


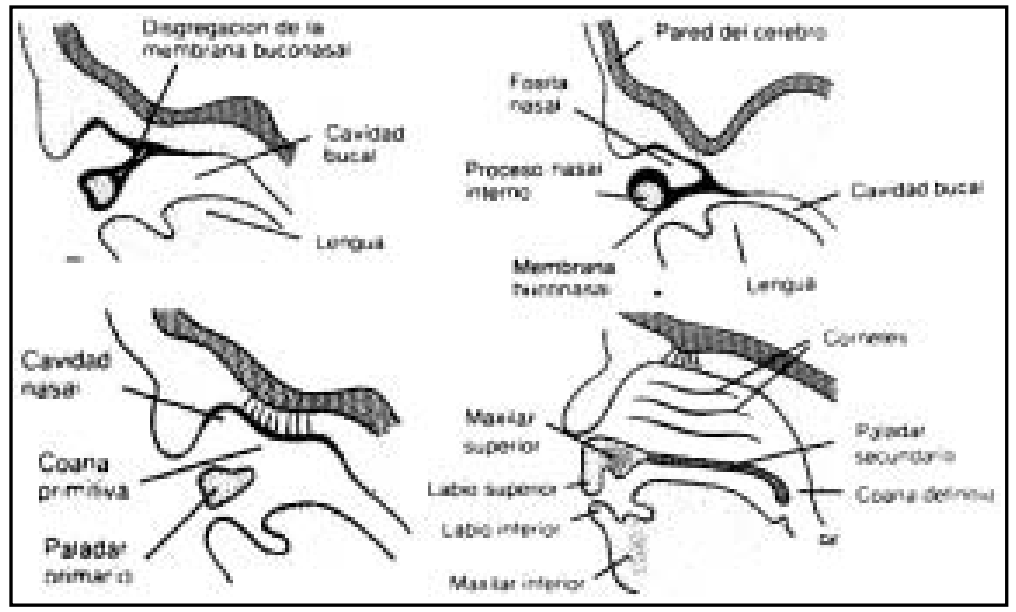

Figura 7. Esquema que muestra la embriología y anatomía de ambas coanas.

Es importante recalcar que el término atresia hace referencia a la imperforación u oclusión de un orificio o conducto normal del cuerpo humano, debiendo ser diferenciado de la "estenosis" que corresponde a la oclusión parcial de algún orificio. Es por esa razón que en estricto rigor el término atresia, debería ser aplicado a las conocidas como "totales" y se debería llamar estenosis a las "parciales".

Tanto la AC adquirida como la estenosis de coanas han sido reportadas en pacientes con el antecedente de uso prolongado de sondas, cirugías otorrinolaringológicas previas (posquirúrgica), cicatrización queloídea y aquellos con carcinoma nasofaríngeo tras ser sometidos a radioterapia ${ }^{2,8}$. Un trabajo realizado por Ku y Tong el año 2006 estudió un total de 7 pacientes con el diagnóstico de carcinoma nasofaríngeo tratados con radioterapia, quienes posteriormente desarrollaron una AC adquirida. La mayoría presentó síntomas tardíamente (más de 6 meses). En relación a los cambios histopatológicos, el denominador común es una reacción inflamatoria severa en la mucosa nasal, la que posteriormente evoluciona con fibrosis del tejido, formando un "diafragma membranoso" en la coana posterior, el cual imita la membrana encontrada en la AC congénita².

En nuestra paciente, la sospecha de una AC adquirida se fundamenta por el antecedente de un uso prolongado de sonda nasoyeyunal y el resultado de la biopsia del tejido comprometido, que mostró la presencia de abundante tejido inflamatorio crónico con extensa fibrosis.

En relación a los síntomas propios de esta anomalía, éstos varían según el grado de compromiso. En los casos unilaterales puede confundirse con otros cuadros obstructivos en los cuales existe obstrucción nasal y rinorrea purulenta unilateral en forma crónica, pasando muchas veces desapercibida por largo tiempo. Cuando el compromiso es bilateral, se manifiesta como un distress respiratorio del recién nacido, con cianosis clínica, la cual característicamente se alivia con el llanto y la alimentación ${ }^{2,3,4}$. En el caso clínico presentado, la paciente presenta obstrucción nasal bilateral progresiva y descarga posterior, que fácilmente podrían ser atribuibles a una patología infecciosa nasosinusal crónica. Esto demuestra que en estos casos la clínica es poco específica, y no existe nada que nos oriente desde un primer momento a hacer un diagnóstico diferencial con una $\mathrm{AC}$ adquirida en un adulto.

El diagnóstico se realiza según el tipo de AC. En un neonato se pueden realizar desde maniobras sencillas, como pasar una sonda nasogástrica durante la atención inmediata del recién nacido, hasta la administración nasal de un colorante, como el azul de metileno lo que nos permite observar su 
paso a través de la nasofaringe, orientándonos al diagnóstico de AC completa.

Por otro lado es importante el estudio mediante imágenes. Entre ellas destacan la tomografía computada de nariz y cavidades paranasales, que actualmente se ha convertido en el examen de elección, y la nasofibroscopía junto a la endoscopía nasal rígida. Esta última, además de confirmar el diagnóstico, nos permite evaluar la localización de la obstrucción, determinar las diferentes características nasales, evaluar la pared lateral nasal y su contribución a la atresia evaluando si se trata de una estenosis ósea y/o membranosa.

En relación al tratamiento, el objetivo primordial de la cirugía es permeabilizar la vía respiratoria nasal y para esto se han descrito múltiples abordajes, cuya utilidad depende de las características del caso y de la experiencia del cirujano ${ }^{6}$. El más utilizado era el transnasal microscópico. Este abordaje tiene la ventaja, gracias al uso del microscopio, de brindar aumento y visión binocular. Para una mejor aproximación se recomienda usar un espéculo nasal autosustentado, el que permite el trabajo con las dos manos $^{11}$. Sin embargo recientemente la Sociedad Americana de Pediatría recomienda el abordaje mediante el uso de endoscopio, con el que se logra un acercamiento más fino. A través de él se dispone de un mejor panorama de la anatomía del paciente, permitiendo tener una mejor noción acerca de los límites del cavum. Las desventajas de esta técnica son la necesidad de personal entrenado y con experiencia en su uso, sobre todo en neonatos. Sin embargo, su utilización es cada vez más frecuente. Nuestra paciente fue sometida a una cirugía endoscópica_a través de la cual se le realizó una septoplastía, y una resección endoscópica de la estenosis, sin incidentes.

Una vez que el paciente es sometido a cirugía, el cirujano enfrenta un nuevo reto frente a la posibilidad de re estenosis, cuyas tasas varían según series, entre un $0 \%$ a $85 \%$. Dado lo anterior, deben tenerse presente varios factores, tanto durante la cirugía como después de ella. Es necesario evitar al máximo el trauma excesivo y realizar una resección adecuada del tabique posterior (vómer) ${ }^{6}$. Se debe proteger la nasofaringe, el paladar blando y la trompa de eustaquio. Para prevenir la estenosis posoperatoria, se usan tutores. Estos últimos no están exentos de complicaciones, como la generación excesiva de tejido de granulación, la perforación septal, la erosión columelar y las infecciones. En el caso reportado, se utilizó un tutor de silicona por ocho semanas, con buena tolerancia, sin presentar complicación alguna.

Por último, también se ha reportado el uso de agentes tópicos que reducen el riesgo de estenosis como la mitomicina-c. Este último es un aminoglicósido que actúa inhibiendo la síntesis de ADN, con lo que impide la proliferación y la migración de fibroblastos, reduciendo la producción de tejido de granulación y ayudando a la cicatrización. Existen estudios que han demostrado su eficacia, al comparar pacientes que no recibieron mitomicina-c con otros que sí lo hicieron. ${ }^{5}$

\section{CONCLUSIONES}

Si bien la atresia de coanas es una malformación frecuentemente congénita, existen factores que aumentan el riesgo de adquirirla en la vida adulta.

En el caso particular de nuestra paciente, el uso prolongado de una sonda nasoyeyunal, se convierte en el eje central de la sospecha de estar frente a una condición atrésica adquirida, ya que la paciente no tiene antecedentes clínicos previos que hagan pensar en un cuadro de otra etiología.

Es importante recalcar, que si bien esta es una condición muy poco frecuente, es necesario siempre tener presente la historia clínica de los pacientes, buscando factores de riesgo, ya sean antecedentes quirúrgicos previos, o el uso prolongado de sondas, como en nuestro caso.

Es importante el adecuado manejo endos-cópico de esta patología, para una resolución óptima.

\section{BIBLIOGRAFÍA}

1. Senders C. Choanal atresia. Otolaryngol Head Neck Surg 2000; 8: 49-53.

2. Ku P, Tong M, Van Hasselt A. Application of holmium yttrium aluminium garnet (YAG) laser in treatment of acquired posterior choanal atresia following radiotherapy for nasopharyngeal carcinoma. The Journal of Laryngology \& Aology 2007; 121: 138-42. 
3. Durón-Martínez J. Atresia de Coanas Bilateral. Reporte de un caso y revisión de la literatura. Rev Med Hond 2004; 72: 21-6.

4. Silva R, Bretón M. Atresia de coanas. Reporte de una caso. Revista Méd 2007; 15: 129-33.

5. Prasad M, Ward R, April M, Bent J, Froehlich P. Topical Mitomicyn as an adjunct to choanal atresia repair. Arch Otolaryng Head and Neck Surg 2002; 128: 398-400.

6. Holland B, McGuirt W. Surgical Management of choanal atresia. Arch. Otolaryng. Head and Neck Surg 2001; 127: 1375-80.

7. Gras A, Paredes 0. Malformaciones congénitas más frecuentes de la vía aérea superior. Pediatr Integral 2005; 9: 181-90.

8. Panda N, Simhadri S, Ghosh S. Bilateral choanal atresia in an adult: is it compatible with life? The Journal of Laryngology \& Aology 2004; 118: 244-5.

9. Duggal P, Kajol L, Duggal K. Unilateral choanal atresia: Presentation in adults. Indian Journal of Aolaryngology and Head and Neck Surgery 2005; 57: 345-7.

10. Herbert $H$. Transnasal mucosal flap rotation technique for repair of posterior choanal atresia. Aolaryngol Head Neck Surg 2001; 124: 674-82. 